\title{
Inhibition of SIRT2 Alleviates Fibroblast Activation and Renal Tubulointerstitial Fibrosis via MDM2
}

\author{
Fang-Fang He ${ }^{a}$ Ren-Yu You ${ }^{b}$ Chen Ye ${ }^{a}$ Chun-Tao Lei ${ }^{a}$ Hui Tang ${ }^{a}$ Hua Su \\ Chun Zhang ${ }^{\mathrm{a}}$
}

aDepartment of Nephrology, Union Hospital, Tongji Medical College, Huazhong University of Science and Technology, Wuhan, bepartment of Radiology, Union Hospital, Tongji Medical College, Huazhong University of Science and Technology, Wuhan, China

\section{Key Words}

Sirtuin 2 • Fibroblast activation • Tubulointerstitial fibrosis • Murine double-minute 2

\begin{abstract}
Background/Aims: Renal tubular epithelial cells and fibroblasts are the main sources of myofibroblasts, and these cells produce the extracellular matrix during tubulointerstitial fibrosis (TIF). Histone deacetylases (HDAC) inhibitors exert an antifibrogenic effect in the skin, liver and lung. Sirtuin 2 (SIRT2), which is a class III HDAC, is an important member of NAD+dependent protein deacetylases. The current study evaluated the role of SIRT2 in renal TIF. Methods: Immunohistochemical staining and Western blot were performed to evaluate SIRT2 expression in TIF patients and unilateral urethral obstruction (UUO) mice. Western blot was used to assess the protein levels of SIRT2, $\alpha$-SMA, collagen III, fibronectin, and MDM2 in tubular epithelial cells and fibroblasts. The specific inhibitor AGK2 was used to inhibit SIRT2 activity, and targeted siRNA was used to suppress SIRT2 expression. Results: SIRT2 expression increased in the tubulointerstitium of TIF patients and UUO mice. SIRT2 inhibition ameliorated TIF in UUO mice. SIRT2 expression in tubular cells was unchanged after exposure to TGF- $\beta 1$. The SIRT2-specific inhibitor AGK2 did not attenuate TGF- $\beta 1$-induced tubular epithelialmesenchymal transition. However, SIRT2 was upregulated in fibroblasts, and fibroblasts were activated after TGF- $\beta 1$ treatment. Genetic knockdown and chemical inhibition of SIRT2 attenuated TGF- $\beta 1$-induced fibroblast activation. We also explored the downstream signaling of SIRT2 during fibroblast activation. Genetic knockdown and chemical inhibition of SIRT2 suppressed TGF- $\beta 1$-induced increase in MDM2 expression, and inhibition of the MDM2-p53 interaction using Nutlin-3 did not suppress SIRT2 upregulation. Conclusion: Our results suggest that SIRT2 participates in the activation of fibroblasts and TIF, which is mediated via regulation of the MDM2 pathway, and the downregulation of SIRT2 may be a therapeutic strategy for renal fibrosis.

(C) 2018 The Author(s) Published by S. Karger AG, Basel
\end{abstract}

F.-F. He and R.-Y. You contributed equally to this work.

Chun Zhang

KARGER
Department of Nephrology, Union Hospital, Tongji Medical College,

Huazhong University of Science and Technology, Wuhan 430022 (China)

Tel. +86-027-85726712, Fax 86-027-83617730, E-Mail drzhangchun@hust.edu.cn 


\section{Cellular Physiology Cell Physiol Biochem 2018;46:451-460

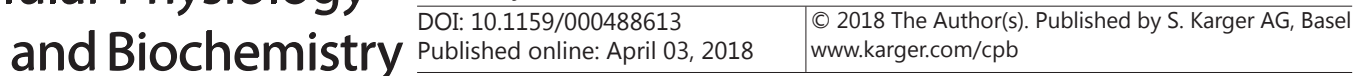 \\ He et al.: SIRT2 in Fibroblast Activation and Renal Tubulointerstitial Fibrosis}

\section{Introduction}

The incidence and prevalence of chronic kidney disease (CKD) increased greatly in recent years and reached 8-16\% worldwide [1]. Many CKD patients progress to end stage renal disease (ESRD), which requires renal replacement therapies. Diabetes, hypertension, and primary glomerulonephritis are the most common causes of CKD. However, the final common pathology of CKD is renal fibrosis, which manifests as glomerulosclerosis and tubulointerstitial fibrosis (TIF) [2]. Renal TIF is characterized by an excessive deposition of extracellular matrix (ECM), inflammatory cell infiltration and myofibroblasts accumulation [3]. Extensive research focused on renal TIF, but the molecular mechanisms still need to be further investigated.

Sirtuin 2 (SIRT2) is a class III histone deacetylase (HDACs) that is associated with metabolic homeostasis [4], neurodegenerative diseases [5], tumorigenesis [6], and inflammatory responses [7]. The early stage of TIF is related to inflammatory responses mediated by infiltrating inflammatory cells, including lymphocytes, macrophages, dendritic cells and mast cells [8]. The inflammatory cells secrete many proinflammatory and profibrotic cytokines, including transforming growth factor- $\beta 1$ (TGF- $\beta 1$ ), which is involved in excessive ECM accumulation and TIF progression [9]. A previous study suggested that SIRT2 promoted hepatic fibrosis, and SIRT2 inhibition attenuated the development of hepatic fibrogenesis [10]. Another study demonstrated that non-specific blockade of the SIRT1/2 pathway attenuated renal fibrosis [11]. Tubular epithelial-mesenchymal transition (EMT) and fibroblast activation are the main mechanisms for TIF progression in kidney. However, the role of SIRT2 in tubular EMT and fibroblast activation still needs to be further clarified.

A previous study reported that the NAD+-dependent protein deacetylase SIRT2 directly deacetylated tumor suppressor p53 in the cytoplasm [12]. Deacetylated p53 inactivates its transcriptional activity, which plays an important role in cellular senescence [13]. An E3ubiquitin ligase murine double-minute 2 (MDM2) also regulates the tumor suppressor p53. MDM2 overexpression promotes ubiquitylation and proteasomal degradation of $\mathrm{p} 53$, which plays a crucial role in tumor progression [14]. Conversely, inhibition of MDM2 re-sensitized rapamycin-resistant renal cancer cells RCC4 and A549 via p53 activation [15]. Another study revealed that MDM2 promoted p53 deacetylation via recruitment of the HDAC1 complex [16]. In kidney, MDM2 is associated with tubular epithelial cell death [17], podocyte mitotic catastrophe [18], kidney development [19] and renal cell carcinoma [20]. Our previous study demonstrated that MDM2 participated in fibroblast activation and TIF via a p53-independent pathway [21]. However, the relationship between SIRT2 and MDM2 in renal TIF is unclear.

The present study investigated the role of SIRT2 in renal TIF. We evaluated SIRT2 expression in human renal TIF tissues and unilateral ureteral obstruction (UUO) mice and investigated the effects of SIRT2 in TGF- $\beta 1$-induced tubular EMT and fibroblast activation. We also examined the downstream molecular mechanism of SIRT2 in TGF- $\beta 1$-induced fibroblast activation.

\section{Materials and Methods}

\section{Human renal biopsy samples}

The Department of Nephrology, Union Hospital, Tongji Medical College, Huazhong University of Science and Technology collected TIF kidney tissue samples from patients with CKD. Control samples were obtained from para-carcinoma renal tissues without any other kidney diseases. All kidney tissues were fixed in 10\% formalin and embedded in paraffin for later use. All patients signed written informed consents. The Ethics Committee of Tongji Medical College, Huazhong University of Science and Technology approved the use of human renal biopsy samples.

Animal experiments

The Animal Care and Use Committee of Tongji Medical College, Huazhong University of Science and Technology approved the animal studies. The UUO model was established in male C57BL/6 mice (Animal 


\section{Cellular Physiology Cell Physiol Biochem 2018;46:451-460 \begin{tabular}{c|c} 
DOI: 10.1159/000488613 & $\begin{array}{l}\text { O 2018 The Author(s). Published by S. Karger AG, Basel } \\
\text { www.karger.com/cpb }\end{array}$
\end{tabular}}

He et al.: SIRT2 in Fibroblast Activation and Renal Tubulointerstitial Fibrosis

Experiment Center, Wuhan University, Hubei, China). All animals had free to access water and a standard normal diet. All experiments were performed on 8-week-old mice weighing 18-22 g. Mice were randomly divided into three groups: sham-operated group; UUO group; and UUO treated with AGK2 (Selleck, Houston, TX, USA) group. Briefly, all animals were anesthetized with $10 \%$ chloral hydrate $(0.5 \mathrm{ml} / 100 \mathrm{~g})$ via intraperitoneal injection. We exposed the abdominal cavity and ligated the left ureter for 7 days. The surgery in the sham group was performed using the same approach to open the abdominal cavity without ligation of the left ureter. AGK2 $(50 \mathrm{mg} / \mathrm{kg})$ was administered via daily intraperitoneal injection for successive 7 days post-surgery. Kidneys were harvested for Western blot analysis and Masson trichrome staining.

\section{Immunohistochemical and Masson trichrome staining}

Immunohistochemical staining was performed on paraffin-embedded kidney tissues. Kidney tissues were deparaffinized, rehydrated, and incubated with an anti-SIRT2 antibody (1:50, Santa Cruz Biotechnology, Santa Cruz, CA, USA) overnight according to a standard protocol. The tubulointerstitial areas were randomly chosen for imaging. The density of positive areas was measured using Image-Pro Plus 6.0 software (Media Cybernetics, Rockville, MD, USA). Masson trichrome staining was performed on paraffin-embedded kidney tissues using standard procedures. The blue color indicated collagen tissue areas, which were quantitatively calculated using ImageJ software (NIH, Bethesda, MD, USA).

\section{Cell culture and treatment}

Rat renal tubular epithelial cells (NRK-52E) were purchased from Western Technology Inc. (Chongqing, China). Rat renal interstitial fibroblasts (NRK-49F) were purchased from American Type Culture Collection (ATCC). Both cell types were cultured in Dulbecco's modified Eagle's medium with F12 (DMEM/F12) containing $10 \%$ fetal bovine serum (FBS) and $100 \mathrm{U} / \mathrm{ml}$ penicillin-streptomycin. Cells were cultured at $37^{\circ} \mathrm{C}$ in a $5 \% \mathrm{CO}_{2}$ humidified incubator. TGF- $\beta 1(5 \mathrm{ng} / \mathrm{ml}$, Proteintech Group, Inc., Wuhan, China) was added to NRK-52E and NRK-49F cells and incubated for the indicated times. Cells were pretreated with AGK2 $(10 \mu \mathrm{M})$ or Nutlin-3 (10 $\mu \mathrm{M}$, Huaxiang Biotechnology Co., LTD., Shanghai, China) for $1 \mathrm{~h}$ prior to TGF- $\beta 1$ treatment.

\section{SIRT2 SiRNA transfection}

Cells were grown to $80 \%$ confluency and transfected with scrambled siRNA and SIRT2 targeted siRNA (RiboBio Co., Ltd., Guangzhou, China) using lipofectamine 2000 (Invitrogen, Carlsbad, CA, USA) for $24 \mathrm{~h}$ according to the manufacturer's instructions. TGF- $\beta 1(5 \mathrm{ng} / \mathrm{ml})$ was added after $24 \mathrm{~h}$ transfection. The efficiency of transfection was evaluated by Western blot.

\section{Western blot analysis}

Kidney tissues and cells were harvested using RIPA buffer (Beyotime Biotechnology, Shanghai, China). The concentrations of harvested proteins were detected using a BCA Protein Assay Kit (Beyotime Biotechnology, Shanghai, China). The same quantity of protein (50 $\mu \mathrm{g}$ ) was loaded onto a sodium dodecyl sulfate (SDS)-polyacrylamide gel for electrophoresis. Proteins were transferred to polyvinylidene difluoride (PVDF, Merck Millipore, Darmstadt, Germany) membranes. Membranes were incubated with 5\% fat-free milk for $1 \mathrm{~h}$ at room temperature and incubated with primary antibodies at $4^{\circ} \mathrm{C}$ overnight. The following primary antibodies were used: SIRT2 (1:400, Santa Cruz Biotechnology, Santa Cruz, CA, USA), $\alpha$-smooth muscle actin ( $\alpha$-SMA, 1:1000, Proteintech Group, Inc., Wuhan, China), collagen III (1:500, Proteintech Group, Inc., Wuhan, China), fibronectin (1:1000, Proteintech Group, Inc., Wuhan, China), MDM2 (1:200, Santa Cruz Biotechnology, Santa Cruz, CA, USA), $\beta$-actin (1:10000, Santa Cruz Biotechnology, Santa Cruz, CA, USA), and GAPDH (1:1000, Abbikine, CA, USA). All membranes were incubated with horseradish peroxidase (HRP)conjugated secondary antibodies (1:5000, Abbikine, CA, USA) for $1 \mathrm{~h}$ at room temperature. Combined antibodies were visualized using an enhanced chemiluminescence detection kit (Merck Millipore, Darmstadt, Germany) and developed on film. All bands were quantified using ImageJ software.

\section{Statistical analysis}

All data are presented as the means \pm SEM. Statistical differences between groups were assessed by one-way analysis of variance (ANOVA). $\mathrm{P}<0.05$ was considered as statistically significant. 


\section{Results}

SIRT2 is elevated in the tubulointerstitium of TIF patients and UUO mice

To evaluate SIRT2 expression in TIF tissues, immunohistochemical staining was performed to detect SIRT2 expression in renal tubulointerstitium of TIF patients and normal control subjects. SIRT2 expression in renal tubulointerstitium was obviously elevated in patients with TIF compared to normal control subjects, especially in the interstitial areas (Fig. 1A-B). SIRT2 expression was markedly elevated in UUO mice compared to sham-operated mice on Western blot analyses (Fig. 1C-D).

\section{Inhibition of SIRT2 activity attenuates TIF in UUO} mice

We administered the specific SIRT2 inhibitor AGK2 to UUO mice to investigate the role of SIRT2 in TIF. Masson trichrome staining revealed tubular dilation, interstitial broadening and increased collagen fibers in UUO mice, and AGK2 administration attenuated collagen deposition in UUO mice (Fig. 2A). Masson trichrome-positive areas were semi-quantitatively analyzed. The results revealed an increase in ECM component collagen in UUO mice compared to shamoperated mice. AGK2 administration decreased collagen in UUO mice compared to UUO mice (Fig. 2B).

TGF- $\beta 1$ does not influence SIRT2 expression, and AGK2 does not attenuate TGF- $\beta 1$-induced EMT and ECM accumulation in cultured tubular epithelial cells

Tubular epithelial cells play critical roles in TIF. To explore the role of SIRT2 in vitro, we treated tubular epithelial cells (NRK-52E) with TGF- $\beta 1$ for 12,24 , and $48 \mathrm{~h}$, and found that SIRT2 protein levels exhibited no significant changes after TGF- $\beta 1$ stimulation compared with the control group using Western blot analysis (Fig. 3A-B). Tubular epithelial cells generally undergo EMT and transform to myofibroblasts that express $\alpha$-SMA and produce excessive ECM after injury [22]. As shown in Fig. 3C-D, TGF- $\beta 1$ increased the expressions of the myofibroblast marker $\alpha$-SMA and ECM components collagen III and fibronectin. However, inhibition of SIRT2 activity by AGK2 did not alleviate TGF- $\beta 1$-induced upregulation of $\alpha$-SMA, collagen III, or fibronectin. These results indicate that SIRT2 is dispensable in TGF- $\beta 1$ induced EMT in tubular epithelial cells.

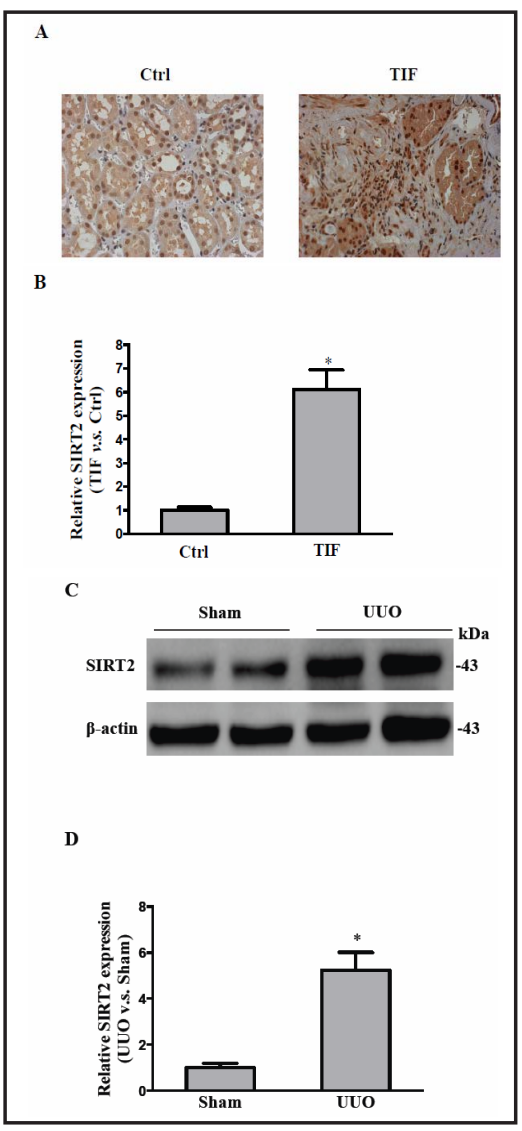

Fig. 1. SIRT2 is elevated in the tubulointerstitium of TIF patients and UUO mice. (A) Immunohistochemical staining of SIRT2 in kidney tissues from Ctrl and TIF patients (original magnification: $\times 400$ ). (B) Summarized data of integrated optical density (IOD)/Area ( $\mathrm{n}=3)$. (C) The protein expression of SIRT2 from Sham and UUO mice kidney tissues were measured by Western blot. (D) Band densities of SIRT2 are expressed as the relative ratio of UUO to Sham $(n=3)$. Ctrl: control samples from para-carcinoma renal tissues without any other kidney diseases; TIF: tubulointerstitial fibrosis kidney tissues from CKD patients. Sham: sham-operated mice; UUO: unilateral ureteral obstruction mice. ${ }^{*} \mathrm{P}<0.05$ vs. Ctrl or Sham.

TGF- $\beta 1$ activates cultured fibroblasts and increases SIRT2 expression in fibroblasts

We clarified the role of SIRT2 in interstitial fibroblast activation. Activated fibroblasts express $\alpha$-SMA and produce excessive ECM components collagen III and fibronectin. Therefore, the expression of $\alpha$-SMA, collagen III and fibronectin was observed in fibroblasts 


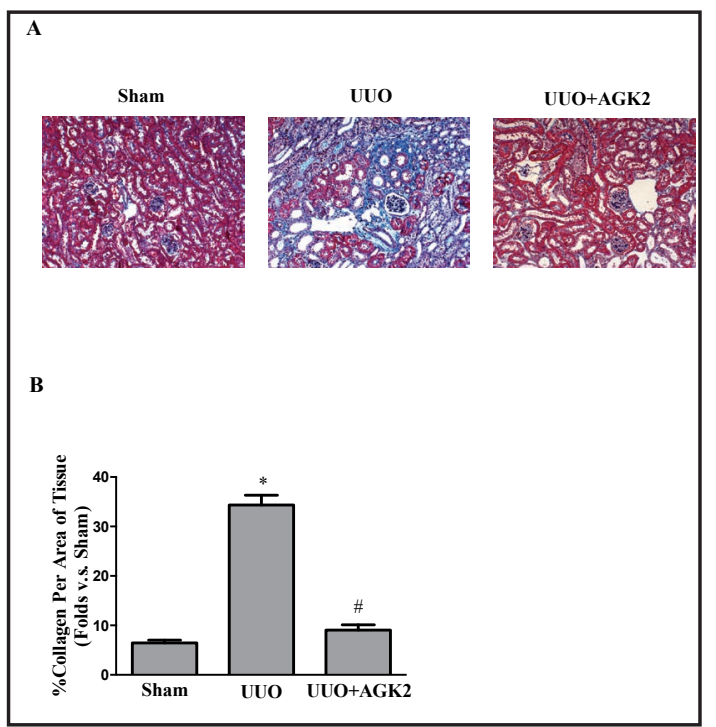

Fig. 2. AGK2 effectively attenuated TIF in UUO mice. (A) Masson trichrome staining of tubulointerstitium areas from Sham, UUO, UUO+AGK2 mice (original magnification: $\times 400$ ). (B) Summarized data of IOD/ Area $(n=4)$. Sham: sham-operated mice; UUO: unilateral ureteral obstruction mice; UUO+AGK2: UUO mice treated with AGK2. ${ }^{*} \mathrm{P}<0.05$ vs. Sham; ${ }^{*} \mathrm{P}<0.05$ vs. UUO.

(NRK-49F) incubated with TGF- $\beta 1$ for 12,24 , and $48 \mathrm{~h}$. The expressions of these three proteins increased significantly after TGF- $\beta 1$ exposure (Fig. 4A-B). TGF- $\beta 1$ stimulation also elevated SIRT2 protein levels. SIRT2 expression was significantly higher at $12 \mathrm{~h}$ and $24 \mathrm{~h}$ and peaked at $24 \mathrm{~h}$ after TGF- $\beta 1$ stimulation (Fig. 4C-D).

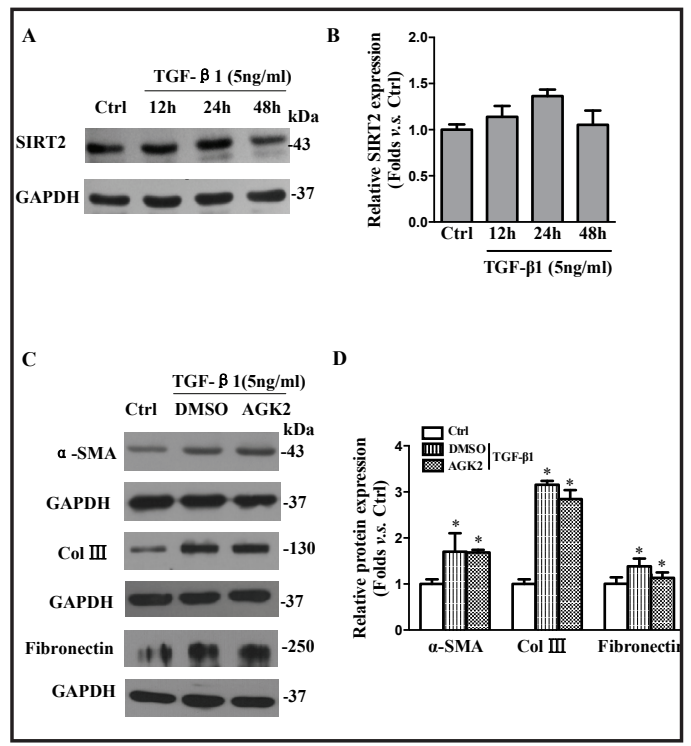

Fig. 3. TGF- $\beta 1$ did not influence SIRT2 expression, and AGK2 did not attenuate TGF- $\beta 1$-induced EMT and ECM accumulation in cultured tubular epithelial cells. (A) The protein expression of SIRT2 in cultured tubular epithelial cells (NRK-52E) was detected by Western blot after TGF- $\beta 1$ exposure for 12,24 , and $48 \mathrm{~h}$. (B) Band densities are presented as the relative ratios of indicated times to Ctrl ( $n=3)$. (C) The protein expressions of $\alpha$-SMA, collagen III and fibronectin in NRK-52E cells were detected by Western blot after exposure to $5 \mathrm{ng} / \mathrm{ml}$ TGF- $\beta 1+D M S O$ or 5 ng/ml TGF- $\beta 1+10 \mu \mathrm{M}$ AGK2. (D) Band densities are presented as the relative ratios of TGF- $\beta 1+$ DMSO or TGF- $\beta 1+A G K 2$ to Ctrl $(n=6)$. Ctrl: control; Col III: collagen III. ${ }^{*} \mathrm{P}<0.05$ vs. Ctrl.

Knockdown of SIRT2 by siRNA transfection attenuates TGF- $\beta 1$-induced fibroblastactivation

To investigate the role of SIRT2 in fibroblast activation, NRK-49F cells were transfected with SIRT2 siRNA or scrambled siRNA for $24 \mathrm{~h}$ then incubated with TGF- $\beta 1$ for $24 \mathrm{~h}$. As shown in Fig. 5A-B, targeting siRNA significantly suppressed SIRT2 protein expression. The protein levels of SIRT2, $\alpha$-SMA, collagen III and fibronectin were significantly lower in SIRT2 siRNA-transfected fibroblasts compared to scrambled siRNA-transfected fibroblasts and untransfected control cells after TGF- $\beta 1$ exposure (Fig. 5C-D).

\section{Inhibition of SIRT2 activity attenuates TGF- $\beta 1$-induced fibroblast activation}

To further examine the role of SIRT2 in fibroblast activation, NRK-49F cells were pretreated with AGK2 for $1 \mathrm{~h}$ and incubated with TGF- $\beta 1$. The expressions of $\alpha$-SMA, collagen III and fibronectin increased significantly in TGF- $\beta 1$-stimulated fibroblasts compared to the control group. AGK2-pretreated fibroblasts exhibited lower expressions of these three proteins compared to fibroblasts stimulated with TGF- $\beta 1$ only (Fig. 6A-B). These results are consistent with the effects of SIRT2 knockdown on fibroblasts and further confirmed the role of SIRT2 in fibroblast activation. 
Fig. 4. TGF- $\beta 1$ induced renal interstitial fibroblast activation and increased SIRT2 expression. (A) The protein expressions of $\alpha$-SMA, collagen III and fibronectin in cultured renal interstitial fibroblasts (NRK-49F) were detected by Western blot after TGF- $\beta 1$ exposure for 12,24 , and 48 h. (B) Band densities are presented as the relative ratios of indicated times to $\mathrm{Ctrl}(\mathrm{n}=3)$. (C) The protein expression of SIRT2 in NRK-49F cells was detected by Western blot after TGF- $\beta 1$ exposure for 12, 24, and $48 \mathrm{~h}$. (D) Band densities are presented as the relative ratios of indicated times to Ctrl ( $n=3)$. Ctrl: control; Col III: collagen III. ${ }^{*} \mathrm{P}<0.05$ vs. Ctrl.

Fig. 5. Downregulation of SIRT2 using targeted siRNA transfection attenuated TGF- $\beta 1$-induced fibroblast activation. (A) The protein expression of SIRT2 after transfection with scrambled and SIRT2 siRNA for $24 \mathrm{~h}$ was measured by Western blot. (B) Band densities of SIRT2 are expressed as the relative ratio of SIRT2 siRNA to scrambled siRNA $(n=3)$. (C) Cells were transfected with scrambled or SIRT2 siRNA for $24 \mathrm{~h}$, and the protein expressions of SIRT2, $\alpha$-SMA, collagen III and fibronectin in NRK-49F cells were detected by Western blot after exposure to $5 \mathrm{ng} /$ $\mathrm{ml}$ TGF- $\beta 1$. (D) Band densities are presented as the relative ratios of different treatments to $\operatorname{Ctrl}(n=6)$. Ctrl: control; Veh: vehicle; Scra: scrambled siRNA; siSIRT2: SIRT2 siRNA. ${ }^{*} \mathrm{P}<0.05$ vs. Ctrl. ${ }^{*} \mathrm{P}<0.05$ vs. TGF- $\beta 1+$ Scra.

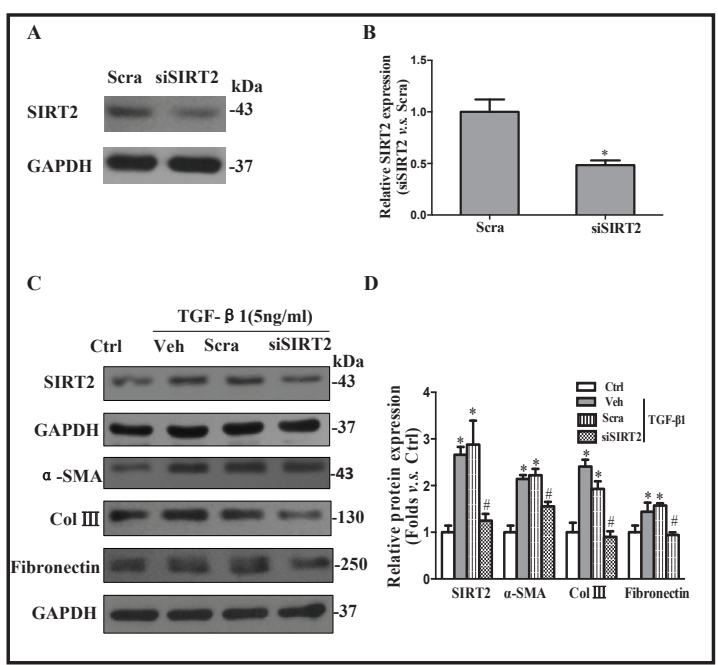

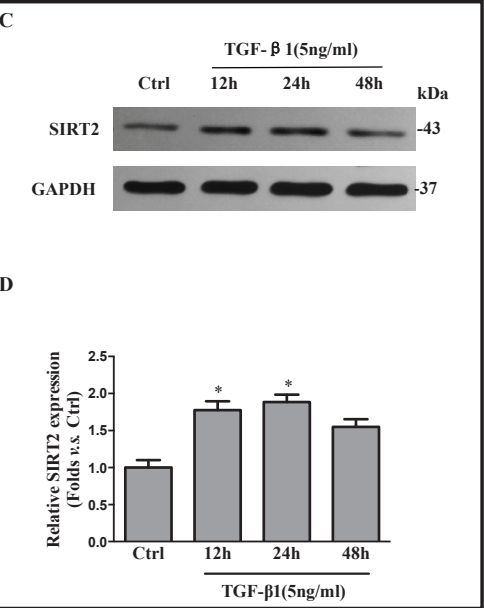

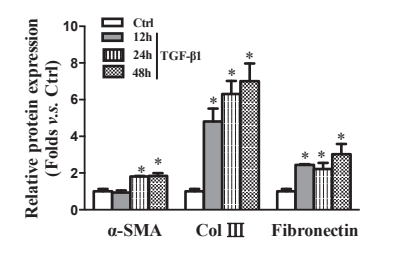

Fig. 6. AGK2 attenuated TGF- $\beta 1$-induced fibroblast activation. (A) The protein expressions of $\alpha$-SMA, collagen III and fibronectin in NRK-49F cells were detected by Western blot after exposure to $5 \mathrm{ng} / \mathrm{ml}$ TGF$\beta 1+$ DMSO or $5 \mathrm{ng} / \mathrm{ml}$ TGF- $\beta 1+10 \mu \mathrm{M}$ AGK2. (B) Band densities are presented as the relative ratios of different treatments to $\mathrm{Ctrl}(\mathrm{n}=6)$. ${ }^{*} \mathrm{P}<0.05$ vs. Ctrl. ${ }^{*} \mathrm{P}<0.05$ vs. TGF- $\beta 1+\mathrm{DMSO}$.

\section{SIRT2 inhibition alleviates TGF- $\beta 1$-induced increase in MDM2 expression}

Previous studies revealed that SIRT2 and MDM2 were involved in the tumor suppressor p53 deacetylation [12, 16]. To investigate the mechanism of SIRT2-mediated fibroblast activation, we detected MDM2 expression after TGF- $\beta 1$ stimulation. As shown in Fig. 7A, TGF- $\beta 1$ stimulation markedly upregulated MDM2. Knockdown of SIRT2 expression using targeted siRNA transfection reduced MDM2 expression compared to the scrambled siRNA 


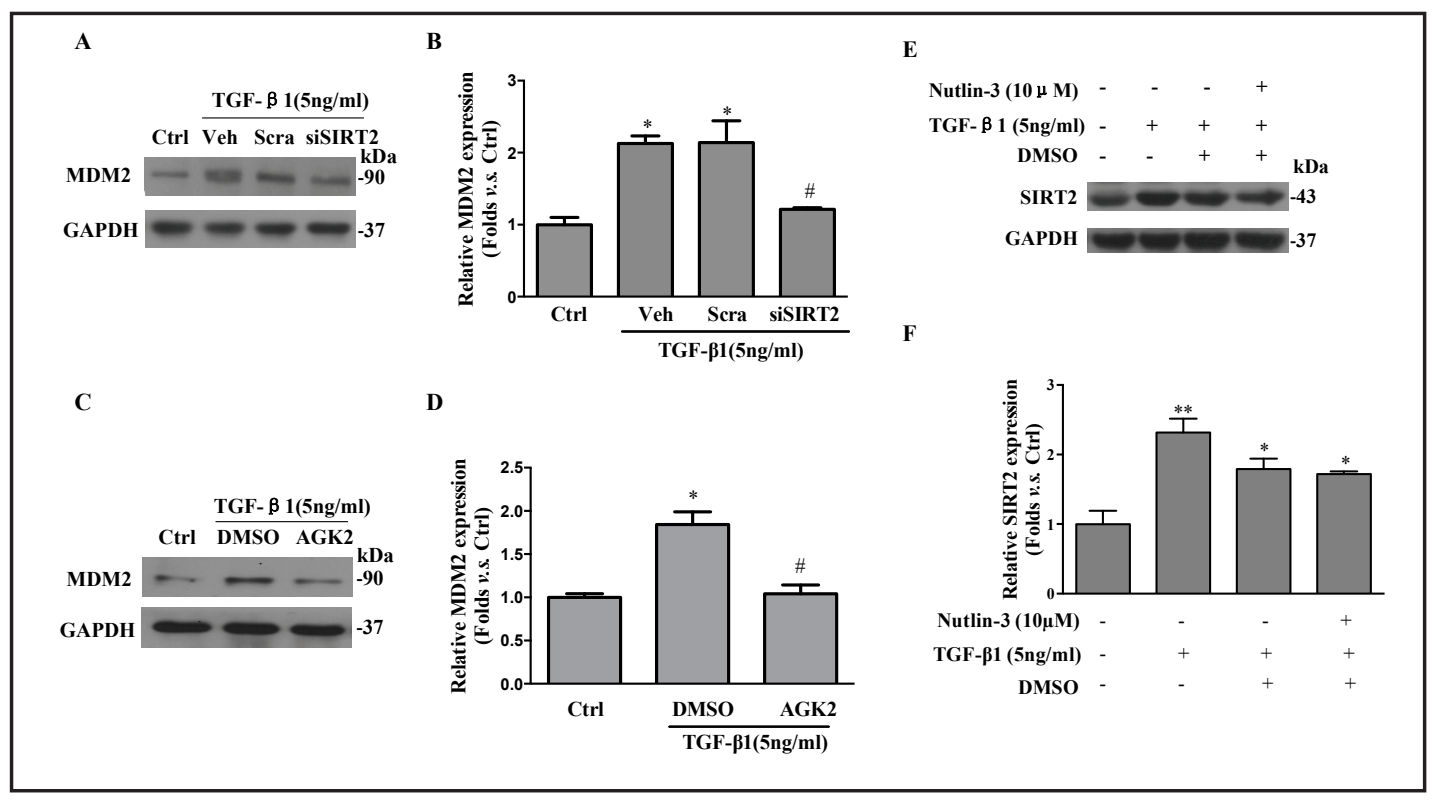

Fig. 7. SIRT2 inhibition alleviated TGF- $\beta 1$-induced increase in MDM2 expression, and Nutlin-3 blockade of the MDM2-p53 interaction did not influence TGF- $\beta 1$-induced SIRT2 upregulation. (A) Cells were transfected with scrambled or SIRT2 siRNA for $24 \mathrm{~h}$, and the protein expression of MDM2 was detected by Western blot after exposure to $5 \mathrm{ng} / \mathrm{ml} \mathrm{TGF- \beta 1.} \mathrm{(B)} \mathrm{Band} \mathrm{densities} \mathrm{are} \mathrm{presented} \mathrm{as} \mathrm{the} \mathrm{relative} \mathrm{ratios} \mathrm{of} \mathrm{different} \mathrm{treat-}$ ments to Ctrl $(n=3)$. (C) The protein expression of MDM2 was detected by Western blot after exposure to 5 $\mathrm{ng} / \mathrm{ml}$ TGF- $\beta 1+$ DMSO or $5 \mathrm{ng} / \mathrm{ml}$ TGF- $\beta 1+\mathrm{AGK} 2$. (D) Band densities are presented as the relative ratios of different treatments to $\mathrm{Ctrl}(\mathrm{n}=3)$. (E) The protein expression of SIRT2 was detected by Western blot after exposure to $5 \mathrm{ng} / \mathrm{ml}$ TGF- $\beta 1+D M S O$ or $5 \mathrm{ng} / \mathrm{ml}$ TGF- $\beta 1+N u t l i n-3$. (F) Band densities are presented as the relative ratios of different treatments to $\mathrm{Ctrl}(\mathrm{n}=3)$. ${ }^{* *} \mathrm{P}<0.01$ vs. $\mathrm{Ctrl}$. ${ }^{*} \mathrm{P}<0.05$ vs. Ctrl. \# $\mathrm{P}<0.05$ vs. TGF$\beta 1+$ Scra or TGF- $\beta 1+$ DMSO

transfection group after TGF- $\beta 1$ treatment (Fig. 7A-B). Inhibition of SIRT2 activity by AGK2 also attenuated TGF- $\beta 1$-induced MDM2 upregulation (Fig. 7C-D). We used the MDM2 and p53 binding inhibitor Nutlin-3 and detected SIRT2 expression by Western blot. Nutlin-3 did not reduce TGF- $\beta 1$-induced SIRT2 upregulation (Fig. 7E-F).

\section{Discussion}

Mammalian SIRTs are a family of $\mathrm{NAD}^{+}$-dependent HDACs that are implicated in a variety of diseases, such as metabolic, age-related, neurodegenerative, cancer and proliferative diseases [23]. There are seven SIRTs, SIRT1-SIRT7, in mammals. SIRT1 is primarily located in the nucleus, and SIRT2 is a cytoplasmic protein. SIRT3, SIRT4 and SIRT5 are primarily located in the mitochondria, and SIRT6 and SIRT7 are predominantly located in the nucleus [24]. This diverse subcellular localization determines their distinct functions. SIRT2 colocalizes with microtubules and deacetylates $\alpha$-tubulin [25], histone H4 substrates and forkhead transcription factor class $O[26,27]$. SIRT2 also interacts with NF- $\kappa$ B family member p65 [28]. Therefore, SIRT2 is involved in many cellular processes, including cell cycle, microvascular inflammation [29], cancer cell proliferation [30], autophagy and apoptosis [31]. Several small molecule inhibitors of SIRTs were reported recently, such as splitomicin and its derivatives, sirtinol, AGK2, cambinol, suramin and tenovin [24]. Class I and II HDACs inhibitors exert anti-inflammatory and anti-fibrotic effects in kidney diseases [32]. Inhibition of the SIRT2 pathway is also anti-fibrotic. A previous study revealed that SIRT2 promoted hepatic fibrosis and SIRT2 inhibition suppressed hepatic fibrogenesis [10]. Jung et al. [33] found that SIRT2 regulated CXCL2 and CCL2 expression in lipopolysaccharide 
(LPS)-induced renal tubular cells injury, and SIRT2 regulation may exhibit beneficial effects in renal inflammatory injury. Another study demonstrated that SIRT2 played a protective role in LPS-induced neuroinflammation [34]. In this study, we found that SIRT2 expression increased significantly in human TIF tissues and the kidney tissues of UUO mice. Inhibition of SIRT2 activity ameliorated renal TIF in UUO mice. These results are consistent with former studies and demonstrate that SIRT2 plays a critical role in inflammation and fibrosis.

The development of renal TIF depends on myofibroblasts because these cells produce a large quantity of ECM components, including collagens and fibronectin. Cellular sources of myofibroblasts generally include resident fibroblasts, tubular epithelial cells, microvascular pericytes, fibrocytes, bone marrow-derived myofibroblasts and endothelial cells [35]. In the tubulointersitium, tubular epithelial cells are the initial site of injury, and then interstitial fibroblasts are activated [9]. Tubular epithelial cells undergo EMT under many pathophysiological conditions [36]. This process promotes tubular epithelial cell transformation into profibrotic myofibroblasts that express the myofibroblast marker $\alpha$-SMA $[37,38]$. Resident fibroblasts are activated and become myofibroblasts expressing $\alpha$-SMA after injury $[39,40]$. TGF- $\beta 1$ is the most important primary driver and mediator during renal interstitial fibrosis. Tubular and interstitial cells synthesize TGF- $\beta 1$. TGF- $\beta 1$ mediates tubular EMT and fibroblast activation, which leads to excessive ECM accumulation and TIF [41]. Our study demonstrated that SIRT2 was expressed in tubular epithelial cells. However, TGF- $\beta 1$ stimulation did not alter SIRT2 expression and the SIRT2 inhibitor AGK2 did not rescue TGF$\beta 1$-induced upregulation of $\alpha$-SMA, collagen III and fibronectin in tubular epithelial cells. These findings differ from another study that demonstrated the involvement of SIRT2 in renal inflammatory injury via regulation of renal tubular CXCL2 and CCL2 expression [33]. These differences may be attributed to the different roles of SIRT2 in renal fibrosis. Then, we used renal interstitial fibroblasts to evaluate the effects of TGF- $\beta 1$. Our results demonstrated that the levels of $\alpha$-SMA, collagen III and fibronectin proteins were increased significantly after TGF- $\beta 1$ treatment in renal interstitial fibroblasts. These results suggest that TGF- $\beta 1$ induces fibroblast activation. We also found that TGF- $\beta 1$ upregulated SIRT2, which was most obvious at $24 \mathrm{~h}$. Downregulation of SIRT2 using siRNA or specific inhibition of SIRT2 using AGK2 suppressed TGF- $\beta 1$-induced fibroblast activation. Therefore, SIRT2 deficiency exerts anti-fibrosis effects via inhibition of fibroblast activation. Taken together, these data suggest that renal interstitial fibroblasts, rather than tubular epithelial cells, are engaged in SIRT2associated renal TIF.

We further explored the molecular mechanism of SIRT2 in TGF- $\beta 1$-induced fibroblast activation. Our previous study demonstrated that MDM2 was also associated with fibroblast activation and TIF progression [21]. The ubiquitin E3 ligase MDM2 is an oncogene that negatively regulates tumor suppressor p53, which is upregulated in a variety of cancer cells [42]. MDM2 reactivated p53 via polyubiquitylation and inhibited its transcriptional activity [43]. Another study demonstrated that SIRT2 deacetylated p53 and inhibited its transcriptional activity [44]. However, the relationship between SIRT2 and MDM2 is not clear. The current study investigated MDM2 expression after TGF- $\beta 1$ stimulation and found that TGF- $\beta 1$ stimulation upregulated MDM2, and SIRT2 inhibition suppressed TGF- $\beta 1$-induced MDM2 upregulation. However, disruption of the MDM2-p53 interaction using Nutlin-3 did not inhibit TGF- $\beta 1$-induced increased SIRT2 expression. These results indicate that SIRT2 is the upstream regulator of MDM2, and it plays important roles in TGF- $\beta 1$-induced fibroblast activation and renal TIF progression via the MDM2 signaling pathway.

\section{Conclusion}

In summary, the present study demonstrated the involvement of SIRT2 in fibroblast activation and renal TIF. SIRT2 inhibition suppressed fibroblast activation via the MDM2 signaling pathway. Therefore, the SIRT2-MDM2 signaling pathway may be a therapeutic target for renal fibrosis.

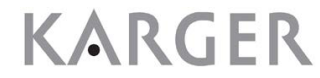




\section{Cellular Physiology Cell Physiol Biochem 2018;46:451-460

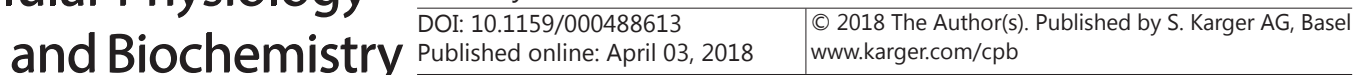

He et al.: SIRT2 in Fibroblast Activation and Renal Tubulointerstitial Fibrosis

\section{Acknowledgements}

This work was supported by grants from the National Natural Science Foundation of China (No. 81522010, No. 81770711, No. 81470964, No. 81570671, No. 81400720, No. 81770736, and No. 81700603) and the Academic Frontier Youth Team Program of Huazhong University of Science and Technology (2016).

\section{Disclosure Statement}

The authors declare that they have no conflicts of interest.

\section{References}

1 Jha V, Garcia-Garcia G, Iseki K, Li Z, Naicker S, Plattner B, Saran R, Wang AY, Yang CW: Chronic kidney disease: global dimension and perspectives. Lancet 2013;382:260-272.

- Webster AC, Nagler EV, Morton RL, Masson P: Chronic Kidney Disease. Lancet 2017;389:1238-1252.

-3 Zeisberg M, Neilson EG: Mechanisms of tubulointerstitial fibrosis. J Am Soc Nephrol 2010;21:1819-1834.

4 Gomes P, Outeiro TF, Cavadas C: Emerging Role of Sirtuin 2 in the Regulation of Mammalian Metabolism. Trends Pharmacol Sci 2015;36:756-768.

5 Donmez G, Outeiro TF: SIRT1 and SIRT2: emerging targets in neurodegeneration. EMBO Mol Med 2013;5:344-352.

-6 Kim HS, Vassilopoulos A, Wang RH, Lahusen T, Xiao Z, Xu X, Li C, Veenstra TD, Li B, Yu H, Ji J, Wang XW, Park SH, Cha YI, Gius D, Deng CX: SIRT2 maintains genome integrity and suppresses tumorigenesis through regulating APC/C activity. Cancer Cell 2011;20:487-499.

-7 Lee AS, Jung YJ, Kim D, Nguyen-Thanh T, Kang KP, Lee S, Park SK, Kim W: SIRT2 ameliorates lipopolysaccharide-induced inflammation in macrophages. Biochem Biophys Res Commun 2014;450:13631369.

8 Razzaque MS, Taguchi T: Cellular and molecular events leading to renal tubulointerstitial fibrosis. Med Electron Microsc 2002;35:68-80.

-9 Tan RJ, Zhou D, Liu Y: Signaling Crosstalk between Tubular Epithelial Cells and Interstitial Fibroblasts after Kidney Injury. Kidney Dis (Basel) 2016;2:136-144.

10 Arteaga M, Shang N, Ding X, Yong S, Cotler SJ, Denning MF, Shimamura T, Breslin P, Luscher B, Qiu W: Inhibition of SIRT2 suppresses hepatic fibrosis. Am J Physiol Gastrointest Liver Physiol 2016;310:G11551168.

11 Ponnusamy M, Zhou X, Yan Y, Tang J, Tolbert E, Zhao TC, Gong R, Zhuang S: Blocking sirtuin 1 and 2 inhibits renal interstitial fibroblast activation and attenuates renal interstitial fibrosis in obstructive nephropathy. J Pharmacol Exp Ther 2014;350:243-256.

12 van Leeuwen IM, Higgins M, Campbell J, McCarthy AR, Sachweh MC, Navarro AM, Lain S: Modulation of p53 C-terminal acetylation by mdm2, p14ARF, and cytoplasmic SirT2. Mol Cancer Ther 2013;12:471-480.

13 Brooks CL, Gu W: The impact of acetylation and deacetylation on the p53 pathway. Protein Cell 2011;2:456462.

14 Meng X, Franklin DA, Dong J, Zhang Y: MDM2-p53 pathway in hepatocellular carcinoma. Cancer Res 2014;74:7161-7167.

15 Tian X, Dai S, Sun J, Jiang S, Sui C, Meng F, Li Y, Fu L, Jiang T, Wang Y, Su J, Jiang Y: Inhibition of MDM2 Re-Sensitizes Rapamycin Resistant Renal Cancer Cells via the Activation of p53. Cell Physiol Biochem 2016;39:2088-2098.

-16 Ito A, Kawaguchi Y, Lai CH, Kovacs JJ, Higashimoto Y, Appella E, Yao TP: MDM2-HDAC1-mediated deacetylation of p53 is required for its degradation. EMBO J 2002;21:6236-6245.

17 Thomasova D, Ebrahim M, Fleckinger K, Li M, Molnar J, Popper B, Liapis H, Kotb AM, Siegerist F, Endlich N, Anders HJ: MDM2 prevents spontaneous tubular epithelial cell death and acute kidney injury. Cell Death Dis 2016;7:e2482.

18 Mulay SR, Thomasova D, Ryu M, Kulkarni OP, Migliorini A, Bruns H, Grobmayr R, Lazzeri E, Lasagni L, 


\section{Cellular Physiology Cell Physiol Biochem 2018;46:451-460 \begin{tabular}{l|l} 
DOI: 10.1159/000488613 & Ond 2018 The Author(s). Published by S. Karger AG, Basel \\
www.karger.com/cpb
\end{tabular}}

He et al.: SIRT2 in Fibroblast Activation and Renal Tubulointerstitial Fibrosis

Liapis H, Romagnani P, Anders HJ: Podocyte loss involves MDM2-driven mitotic catastrophe. J Pathol 2013;230:322-335.

19 El-Dahr S, Hilliard S, Aboudehen K, Saifudeen Z: The MDM2-p53 pathway: multiple roles in kidney development. Pediatr Nephrol 2014;29:621-627.

20 Noon AP, Vlatkovic N, Polanski R, Maguire M, Shawki H, Parsons K, Boyd MT: p53 and MDM2 in renal cell carcinoma: biomarkers for disease progression and future therapeutic targets? Cancer 2010;116:780-790.

21 Ye C, Tang H, Zhao Z, Lei CT, You CQ, Zhang J, Gao P, He FF, Chen S, Wang YM, Zhang C, Su H: MDM2 mediates fibroblast activation and renal tubulointerstitial fibrosis via a p53-independent pathway. Am J Physiol Renal Physiol 2017;312:F760-768.

-22 Liu Y: New insights into epithelial-mesenchymal transition in kidney fibrosis. J Am Soc Nephrol 2010;21:212-222.

-23 Yamamoto H, Schoonjans K, Auwerx J: Sirtuin functions in health and disease. Mol Endocrinol 2007;21:1745-1755.

24 Villalba JM, Alcain FJ: Sirtuin activators and inhibitors. Biofactors 2012;38:349-359.

25 North BJ, Marshall BL, Borra MT, Denu JM, Verdin E: The human Sir2 ortholog, SIRT2, is an NAD+dependent tubulin deacetylase. Mol Cell 2003;11:437-444.

-26 Vaquero A, Scher MB, Lee DH, Sutton A, Cheng HL, Alt FW, Serrano L, Sternglanz R, Reinberg D: SirT2 is a histone deacetylase with preference for histone H4 Lys 16 during mitosis. Genes Dev 2006;20:1256-1261.

27 Li W, Zhang B, Tang J, Cao Q, Wu Y, Wu C, Guo J, Ling EA, Liang F: Sirtuin 2, a mammalian homolog of yeast silent information regulator-2 longevity regulator, is an oligodendroglial protein that decelerates cell differentiation through deacetylating alpha-tubulin. J Neurosci 2007;27:2606-2616.

-28 Rothgiesser KM, Erener S, Waibel S, Luscher B, Hottiger MO: SIRT2 regulates NF-kappaB dependent gene expression through deacetylation of p65 Lys310. J Cell Sci 2010;123:4251-4258.

-29 Buechler N, Wang X, Yoza BK, McCall CE, Vachharajani V: Sirtuin 2 Regulates Microvascular Inflammation during Sepsis. J Immunol Res 2017;2017:2648946.

30 Du Y, Wu J, Zhang H, Li S, Sun H: Reduced expression of SIRT2 in serous ovarian carcinoma promotes cell proliferation through disinhibition of CDK4 expression. Mol Med Rep 2017;15:1638-1646.

-31 Kim MG, Kim DH, Lee HR, Lee JS, Jin SJ, Lee HT: Sirtuin inhibition leads to autophagy and apoptosis in porcine preimplantation blastocysts. Biochem Biophys Res Commun 2017;488:603-608.

-32 Brilli LL, Swanhart LM, de Caestecker MP, Hukriede NA: HDAC inhibitors in kidney development and disease. Pediatr Nephrol 2013;28:1909-1921.

33 Jung YJ, Lee AS, Nguyen-Thanh T, Kim D, Kang KP, Lee S, Park SK, Kim W: SIRT2 Regulates LPS-Induced Renal Tubular CXCL2 and CCL2 Expression. J Am Soc Nephrol 2015;26:1549-1560.

-34 Wang B, Zhang Y, Cao W, Wei X, Chen J, Ying W: SIRT2 Plays Significant Roles in LipopolysaccharidesInduced Neuroinflammation and Brain Injury in Mice. Neurochem Res 2016;41:2490-2500.

-35 Sun YB, Qu X, Caruana G, Li J: The origin of renal fibroblasts/myofibroblasts and the signals that trigger fibrosis. Differentiation 2016;92:102-107.

-36 Wang B, Ding W, Zhang M, Li H, Gu Y: Rapamycin attenuates aldosterone-induced tubulointerstitial inflammation and fibrosis. Cell Physiol Biochem 2015;35:116-125.

-37 Quaggin SE, Kapus A: Scar wars: mapping the fate of epithelial-mesenchymal-myofibroblast transition. Kidney Int 2011;80:41-50.

-38 Hu J, Zhu Q Li PL, Wang W, Yi F, Li N: Stem cell conditioned culture media attenuated albumin-induced epithelial-mesenchymal transition in renal tubular cells. Cell Physiol Biochem 2015;35:1719-1728.

-39 Grande MT, Lopez-Novoa JM: Fibroblast activation and myofibroblast generation in obstructive nephropathy. Nat Rev Nephrol 2009;5:319-328.

40 Di J, Jiang L, Zhou Y, Cao H, Fang L, Wen P, Li X, Dai C, Yang J: Ets-1 targeted by microrna-221 regulates angiotensin II-induced renal fibroblast activation and fibrosis. Cell Physiol Biochem 2014;34:1063-1074.

41 Lopez-Hernandez FJ, Lopez-Novoa JM: Role of TGF-beta in chronic kidney disease: an integration of tubular, glomerular and vascular effects. Cell Tissue Res 2012;347:141-154.

42 Zhao Y, Yu H, Hu W: The regulation of MDM2 oncogene and its impact on human cancers. Acta Biochim Biophys Sin (Shanghai) 2014;46:180-189.

43 Wang X, Jiang X: Mdm2 and MdmX partner to regulate p53. FEBS Lett 2012;586:1390-1396.

-44 Jin YH, Kim YJ, Kim DW, Baek KH, Kang BY, Yeo CY, Lee KY: Sirt2 interacts with 14-3-3 beta/gamma and down-regulates the activity of p53. Biochem Biophys Res Commun 2008;368:690-695. 